\title{
Herramientas multimedia que mejoran el aprendizaje integral del electromagnetismo
}

\author{
Francisco S. Barralaga V. ${ }^{1}$ y Alejandro Galo Roldán ${ }^{2}$ \\ ${ }^{1}$ Escuela de Física - UNAH, mail: fbarralaga@gmail.com \\ ${ }^{2}$ Escuela de Física - UNAH, mail: alejandrogaloroldan@gmail.com
}

Recibido: 14 de Diciembre de 2013 / Aceptado: 30 de Julio de 2016

\begin{abstract}
Resumen
The difficulties inherent to electromagnetism topics study, have motivated an increasing activity in the educational institutions of the world focused in develop options that generate, in students, significant improvements in the process of learn these subjects. These tools make more and more extensive use of the multimedia facilities provided by information and computer technology. A wide variety of hardware tools have been developed in the field of physics, many use of expensive computer resources, but there are some that have been implemented with multimedia or programming resources that are freely available to the world on open source resource platforms. This article is the result of a recent bibliographical research on trends in the teaching of the physical sciences in the world, particularly in the field of electromagnetism. Some important implementations for Windows environments that were found in the process of this research are discussed, in order to indicate their availability and motivate their use to develop and implement their own legal options that help solve the difficulties presented by our Students of science and engineering in the integral assimilation of the concepts of Electromagnetic Theory.
\end{abstract}

Keywords: Physics Teaching, Computer Simulation, Online help, Own didactic developments, Open Source Tools

\begin{abstract}
Las dificultades inherentes al estudio de tópicos de electromagnetismo, han motivado una creciente actividad en las instituciones educativas del mundo dirigida a desarrollar opciones que generen en los estudiantes, mejoras significativas en los niveles de aprendizaje de estos temas. Estas herramientas hacen uso, en forma cada vez más extensiva, de las facilidades multimedia que brindan las tecnologías de información y computación. Se ha desarrollado una gran variedad de herramientas didácticas en el campo de la física, muchas hacen uso de recursos computacionales costosos, pero existen algunas que han sido implementadas con recursos multimedia o de programación que están disponibles al público en forma gratuita en plataformas de recursos de código abiertø 1 Este artículo es el resultado de una investigación bibliográfica reciente sobre las tendencias de la enseñanza de las ciencias físicas en el mundo, particularmente en el campo del electromagnetismo. Se discuten algunas implementaciones importantes para ambientes Windows que fueron encontradas en el proceso de esta investigación, a fin de señalas su disponibilidad y motivar su uso para desarrollar e implementar opciones didáctica propias que ayuden a resolver las dificultades que presentan nuestros estudiantes de ciencias e ingeniería en la asimilación integral de los conceptos de la Teoría Electromagnética.
\end{abstract}

Palabras clave: Enseñanza de la Física, Simulación en computadora, Ayudas en línea, Desarrollos didácticos propios, Herramientas de Código Abierto

\section{INTRODUCCIÓN}

厂 Os estudiantes que inician su estudio de la Teoría Electromagnética, evidencian dificultades y concepciones erróneas, en particular cuando se abordan las conceptos acerca de las fuentes de los campos electromagnéticos 14. Son conocidas las dificultades que afrontan los estudiantes de ingeniería [23], en el estudio de campos electromagnéticos variantes con el tiempo, una temática imprescindible en el estudio de tópico de antenas y comunicaciones inalámbricas, y por demás esencial para desarrollar respuestas tecnológicas adecuadas requeridas en el área de las telecomunicaciones. La Teoría Electro- magnética es una temática que forma parte del pensum académico de cualquier carrera de ciencias o de ingeniería siendo una componente fundamental de la formación teórica básica de los estudiantes de estas carreras.

Mucho esfuerzo se ha puesto en desarrollar opciones auxiliares educativas, específicamente en el área de electromagnetismo reportándose en todos los casos mejoras en el rendimiento académico de los estudiantes.

Durante la primera década del siglo veintiuno, en varios países del mundo, fueron implementadas ayudas en línea, se diseñaron y construyeron equipo de adquisición de datos, software para análisis y creación de modelos a 
partir de datos colectados, aplicaciones desarrolladas en lenguajes de computación y sistemas basados en interfaces gráficas de usuario, todas enfocadoas en el estudio de fenómenos físicos (incluyendo electromagnetismo) basados en simulación.

En el ambiente tecnológico de la Universidad Nacional Autónoma de Honduras (UNAH), permite que muchos procesos docentes sean realizados a través del Internet. El estudiante que actualmente ingresa al área físico-matématica, tiene habilidades suficientemente desarrolladas en manejo de TIC, como para usar con autonomía recursos disponibles en la Web, incluyendo la manipulación de texto, imágenes, sonido y vídeo, mismos que pueden incorporarse a sistemas de enseñanza virtuales que redunden en ventajas útiles para integrar el conocimiento del electromagnetismo y otros campos del conocimiento.

\section{Desarrollo del Tema}

La experiencia de muchos años de labor docente de la Escuela de Física de la UNAH, evidencia una creciente dificultad que afecta a los alumnos y alumnas que cursan las asignaturas de física incluidas en el pensum de carreras de ciencias e ingeniería. Esto plantea una problemática cuya solución debe ser buscada.

En países como Honduras, el acceso a equipo de laboratorio es muy limitado o inexistente, y el estudio de la física, una ciencia experimental, se vuelve un reto al reducir las opciones de integración y asimilación de los conceptos físicos, a la capacidad de abstracción de los estudiantes.

Las dificultades no se reducen al limitado acceso a laboratorios experimentales de ciencias físicas. Recientemente se ha planteado 9] como un problema, la actitud que presentan los estudiantes de física al no leer sus libros de texto. Los autores usaron módulos basados en sistemas multimedia ubicados en sitios web, para exponer a un grupo grande de estudiantes de física a pre-lecturas que eran requeridas para completar su preparación inicial a la lectura de un artículo referencial. Ellos aseguran que no es una estrategia efectiva, el que las lecturas previas asignadas se hagan solo de los libros de texto.

"Todo problema tiene al menos una solución". Eso dice una conocida frase, a la que se hace alusión cuando se encara una problemática que necesita ser resuelta. En este contexto, se ha realizado una investigación bibliográfica sobre los problemas de aprendizaje de la física detectados en instituciones educativas del mundo, y sobre las estrategias que estas han usado para intentar resolverlos. Fueron consultadas fuentes internacionales de investigación en el tema de la enseñanza de las ciencias físicas incluidas en las bases de datos para la investigación señaladas por el Sistema Bibliotecario de la UNAH. Los artículos, citados en todo el documento e incluidos en la bibliografía al final del mismo, fueron analizados y los resultados sirven de marco teórico de esta publicación.
Se mencionan en este documento las herramientas que han sido reportadas en publicaciones dedicadas a la enseñanza de las ciencias físicas, por instituciones educativas de nivel superior de varios países del mundo. El objetivo es provocar la reflexión de la comunidad docente del área físico-matemática alrededor de los problemas de rendimiento académico de nuestros estudiantes y contribuir al inicio del proceso de búsqueda de nuestras propias soluciones.

La investigación realizada mostró que los recursos educativos desarrollados en un contexto multimedia, han tenido un importante y positivo impacto en la enseñanza de la física. Estas implementaciones estimulan la memoria audio-visual y transportan al estudiante al centro mismo de los fenómenos [6].

En varios países de Europa [8] se utilizan sistemas multimedia en clases y en laboratorios para complementar los procesos de enseñanza tradicional con la simulación gráfica de los fenómenos físicos, y el modelamiento con programas de computadora especializados en análisis gráfico de los datos experimentales tomados en las sesiones de laboratorio experimental.

Un estudio realizado en el 2009 en Grecia 26] sobre la mejora en el aprendizaje de las ciencias naturales en estudiantes de últimos años de secundaria, reportó resultados significativos cuando se hizo uso de simulaciones con despliegues gráficos bidimensionales y tridimensionales.

\section{ENSENANANA DEL ELECTROMAGNETISMO}

Las dificultades particulares que enfrenta la enseñanza del electromagnetismo, requieren de ayudas o auxiliares de estudio individual, que permitan la visualización de los campos eléctricos y magnéticos en sus procesos dinámicos de interacción. Los niveles de abstracción necesarios, la matemática involucrada, y las limitaciones del tiempo de duración de los cursos, no dan lugar a la intervención plena de metodologías constructivas.

Según reportan investigadores de la Universidad de Murcia 14, hasta antes del 2004 muy poca investigación se había realizado acerca de la enseñanza del electromagnetismo a nivel de pregrado y posgrado. La mayoría de las investigaciones en el campo, realizadas antes de este año, se enfocaban más bien al nivel de secundaria. Los estudios realizados a nivel universitario, a finales de los años 90, [3, [5, 10, 13, 18, 19], revelaron que la mayoría de los estudiantes, después de que un largo periodo de instrucción, no manifiestan un aprendizaje significativo de la teoría de campos electromagnéticos. Recientes estudios indican lo mismo[27].

Hay implementaciones para mejorar la actitud de los estudiantes hacia las lecturas de temas de física, con la disposici ón de sitios web dedicados a lectura [9] y se considera el uso de herramientas de simulación en computadora para obtener las visualizaciones de los campos 
electromagnéticos 20, 21, 26] e incluso se registran experiencias en las que han sido usadas herramientas de modelamiento de datos experimentales colectados por los mismos estudiantes en sesiones de laboratorio [11, 21].

En todos estos estudios quedan claramente evidenciadas las dificultades que presentan los estudiantes de nivel universitario, en los conceptos inherentes a los campos magnéticos y eléctricos. Las soluciones propuestas por estos investigadores han sido variadas, pero ciertamente todos coinciden en que la asistencia de las computadoras y de las tecnologías de información (TIC) es fundamental.

\section{MATLAB Y MATHCAD}

La interface y las opciones gráficas de Matlab ${ }^{2}$ son ampliamente utilizadas para desarrollar herramientas educativas de software para la visualización y el análisis de los fenómenos de propagación de ondas electromagnética [7, 23].

A través de estas interfaces, es posible que los estudiantes manipulen los parámetros relacionados con las expresiones matemáticas de los fenómenos y que visualicen los efectos de estos cambios en el comportamiento simulado en computadora. Una ventaja adicional de Matlab, es que permite el ingreso de datos experimentales para modelarlos numéricamente y para comparar los resultados con los modelos teóricos estudiados en clase.

Aunque en menor grado pero igualmente importantes que los desarrollos en Matlab, son las aplicaciones que incluyen simulaciones de fenómenos electromagnéticos desarrollados con MathCAD ${ }^{3} 21$.

Por ser herramientas comerciales, estos sistemas tienen costos altos, tendiendo a ser más sofisticadas y complejas; para mejorar el desarrollo de sus aplicaciones, se concentran en dar los resultados de los cálculos involucrados, dejando prácticamente ocultos los detalles de los procesos numéricos, las manipulaciones de las ecuaciones y de los parámetros que constituyen los modelos de los fenómenos estudiados [20]; esto reduce las posibilidades de generar mayor grado de conocimientos significativos en los estudiantes.

\section{A. Cursos en Línea}

Otra manera importante en la que se desarrollan opciones educativas para la enseñanza del electromagnetismo, son los cursos en línea en la "web" 77 a los que los estudiantes acceden por la internet ("e-Courses" como son denominados en inglés). En estos se solicita a los alumnos, el estudio del material dispuesto en los sitios que se instalan en servidores con plataforma Moodle (opción

\footnotetext{
${ }^{2}$ Conocido sistema de cálculo numérico y álgebra simbólica creado y mercadeado por la compañía Mathworld. Su nombre es la combinación de las primeras letras de Matrix Laboratory, "laboratorio de matrices".

${ }^{3}$ Sistema de manipulación y análisis numérico y simbólico distribuido por PTC.
}

que está disponible en la UNAH) que permite el monitoreo del tiempo de estudio individual de cada estudiante, información que puede ser utilizada tanto para fines de evaluación como para fines de investigación educativa.

\section{RECURSOS LIBRES}

Es importante considerar recursos gratuitos que puedan ser usados para la creación de material para fines educativos. Productos como Matlab, MathCAD, Simulink, LabView, y otros, representan atractivas interfaces con opciones gráficas, con las que pueden crearse simulaciones y modelamiento de fenómenos electromagnéticos, pero implican el consumo de recursos financieros necesarios para la compra de las licencias correspondientes, recursos, que no suelen estar siempre disponibles en Honduras.

En este sentido mencionaré los reportes (con muy buenos resultados) del uso de Java ${ }^{4}$ en la creación de simulaciones de la temática de la Teoría Electromagnética [11, 15]. Algunas de estas aplicaciones creadas con Java (denominados Applets), están disponibles en los sitios "web" de los autores, a los que hay que acceder para su utilización, y han sido parte de varias implementaciones educativas no solo en el campo del electromagnetismo.

\section{SCILAB}

Algunas de estas últimas, como Scilah 5 tiene un alto grado de sostificación y requieren de mayor tiempo de elaboración [16.

\section{EASY JAVA SIMULATION}

Por otra parte, se vinculan en la documentación analizada, a algunas herramientas gratuitas [4, 12, 24], que tienen la particularidad de interactuar con los modelos matemáticos y asá generar mayor nivel de constructivismo.

Una importante herramienta gratituita a la que se hace referencia en los artículos, es EJS ${ }^{6}$ (Easy Java Simulations). EJS es un software basado en Java 7 que genera simulaciones de fenómenos físicos de buena calidad consumiendo poco tiempo de implementación[12, y representa una solución para ambientes académicos con recursos limitados como la Escuela de Física de la UNAH.

Easy Java Simulations, es una herramienta diseñada para la creación de simulaciones interactivas de fenómenos científicos en computadora. Las simulaciones en computadora se usan para explicar mejor los conceptos más

\footnotetext{
${ }^{4}$ Java es un lenguaje gratuito creado por Sun Electronics, que puede ser descargado de internet.

${ }^{5}$ Scilab (Scientific Laboratory), es una herramienta de simulación y modelaje gratuita. http//www.scilab.org/

${ }^{6}$ EJS (Easy Java Simulations): http://fem.um.es/Ejs/

${ }^{7}$ Java es un lenguaje gratuito, ampliamente utilizado en dispositivos digitales creado por Sun Electronics.
} 
difíciles, y permitir a los estudiantes trabajar con los modelos ya desarrollados incluidos en ellas, pudiéndose aun, después de una breve capacitación básica, permitir a los estudiantes la creación de sus propias aplicaciones.

Easy Java Simulations, es totalmente gratuita y puede fácilmente ser descargada de su sitio web oficial; ha sido ampliamente utilizada para el análisis visual y numérico de modelos de física simulados en computadora. Fue desarrollada como parte de uno de los proyectos educativos más ambiciosos llevados a cabo en Europa en la última década [11], por un grupo de once países de este continente, entre ellos España, Italia y Holanda.

EJS fue creada por Francisco Esquembre, un físico español de la Universidad de Murcia, y es parte de un reciente proyecto europeo de "código abierto" (Open Source en inglés) dirigido al estudio del electromagnetismo y la superconductividad 12. Cuenta con opciones para interactuar con Moodle, y recientemente han creado un app ${ }^{8}$ para poder correr las aplicaciones en tablet 9 lo que la hace especialmente atractiva para el desarrollo de aplicaciones en línea instaladas en esta plataforma educativa también gratuita.

\section{Plataforma IDEAL PARA ESTUdiO DE CIENCIAS FÍSICAS}

Una estructura de aprendizaje ideal para la enseñanza de estos tópicos, debería disponer de montajes experimentales con dispositivos especiales para la detección de las señales producidas por los fenómenos físicos, integrados a sistemas de adquisición de datos con opciones de modelamiento matemático auxiliado por computadora, contando además, con herramientas de simulación de estos fenómenos para el estudio detallado de los conceptos; tal herramientas es ciertamente costosa, pero debería ser el objetivo al que aspire toda unidad de enseńanza de la física a nivel universitario.

En este sentido se reporta el desarrollo del Proyecto MOSEM[11], dirigido a escuelas secundarias, con una importante componente de recolección y análisis de datos experimentales a través de interfaces de adquisición de datos (o "Dataloggers"), conectadas a una gran variedad de detectores de señales físicas; su segunda componente importante lo constituye el modelamiento matemático de esta información experimental, y su tercer componente, con el que se completa la opción educativa, consiste en el uso de simulaciones creadas con EJS, para mejorar el aprendizaje de fenómenos físicos.

\footnotetext{
${ }^{8}$ Abreviatura de application package.

${ }^{9}$ Dispositivos ligeros inicialmente diseñados para la lectura digital cuyas características actuales y bajo costo, les confieren ciertas ventajas sobre las Laptops.
}

\section{CONCLUSIONES}

Los investigadores de la enseñanza de las ciencias físicas en varios países, coinciden en que el estudio de este campo del conocimiento tanto a nivel secundario como universitario, plantea dificultades importantes para los y las estudiantes. El problema se vuelve más evidente con los tópicos incluidos en la Teoría Electromagnética.

Los investigadores proponen el uso de implementaciones que consideren opciones multimedia, o ayudas asistidas por computadora con simulaciones de los fenómenos estudiados en física. En todos los artículos se reportan mejoras en el rendimiento de los grupos de control que fueron expuestos a estas herramientas.

Han sido ampliamente utilizados para desarrollar herramientas complementarias del estudio de la Teoría Electromagnética, sistemas computacionales de alto costo, tales como MatLab y MathCAD, pero se encontraron también recursos gratuitos de código abierto, como Java, Easy Java Simulation y Scilab, que pueden ser considerados como opciones de desarrollo de herramientas didácticas para nuestros estudiantes de ciencias e ingeniería.

El uso de la plataforma Moodle, es reportada también como una herramienta útil para el desarrollo de opciones auxiliares en línea que puedan ser de ayuda en el estudio de las ciencias físicas.

\section{REFERENCIAS}

[1] Aranzabal, J. (2003). Dificultades de aprendizaje de los estudiantes universitarios en la teoría del campo magnético y elección de los objetivos de enseñanza.

[2] Aranzabal, J. (s.f.). Campo magnético: diseño y evaluación de estrategias de enseñanza basadas en el aprendizaje como investigación orientada. Enseñanza de las ciencias: revista de investigación y experiencias didácticas, 23(3), 303-319.

[3] Bagno, E. (1997). From problem solving to a knowledge structure: An example from the domain of electromagnetism. American Journal of Physics, 726736.

[4] Benavides, G. A. (2011). Resources for an electronic engineering undergraduate program. Latin American and Caribbean Journal of Physics, 5(2).

[5] Borges, A. (1998). Models of magnetism. international journal of science edication. International Journal of Science Education, 20(3), 361-378.

[6] Bostan, C. (2006). The modern aids used to teach physics: Computer-assited instruction. Analele Universutatii din Bucuresti, Fisica-LV.

[7] Branislav Korenko, J. (2010). Teaching of electromagnetism using kenya software. Joint International IGIP-SEFI Anual Conference 19th. Trnava, Slovakia. 
[8] Carmen, G. (2011). Modelling physic's experiments with kenya software. 7a. Conferencia Cientifica Internacional de Educación Electrónica y Software Educativo.

[9] Chen, Z.; Stelzer, T. y Gladding, G. (2010). Using multimedia modules to better prepare students for introductory physics lecture. Phys Rev ST Phys Educ Res, 6, doi:10.1103/physrevstper. 6.010108. URL http://gen.lib.rus.ec/scimag/ index.php?s=10.1103/physrevstper.6.010108.

[10] Colombo de Cudmani, L. . (1990). Concepciones previas en el aprendizaje significativo del electromagnetismo. Enseñanza de las Ciencias, 8(3), 215-222.

[11] E.Kedzierska(1), V. S. (2011). Mosem project. Integration of data acquision, modelling, simulation and animation for learning electromagnetism and supercondutivity. Europa:(1)Universidad de Amsterdam, Holanda; (2)Simplicatus AS, Noruega; (3)University of Murcia, España; (4)Departamento de Física de la Universidad de Ostrava, (5)República Checa; (6)Departamento de Fí sica de la Universidad de Udine, Italia.

[12] Esquembre, F. (2004). Easy java simulations: a software tool to create scientific simulations in java. Computer Physics Communications, 199-204.

[13] Galili, I. (1997). Changing approach to teaching electromagnetism in a conceptually oriented introductory physics course. American Journal of Physics, 65(7), $657-667$.

[14] Guisasola, J.; Almudí, J. M. y Zubimendi, J. L. (2004). Difficulties in learning the introductory magnetic field theory in the first years of university. Science Education, 88, doi:10.1002/sce.10119.

[15] Hargunani, S. P. (2010). Teaching of faraday's and lenz's theory of electromagnetic induction using java based faraday's lab simulations. Latin American Journal of Physics Education, 4(3).

[16] Longhua Ma, F. X. (2008). Integrated design and implementation of embedded control systems with scilab. Sensors.

[17] Marti, A. U. (2007). Aplicación de la simulación por ordenador a la enseñanza de las ciencias. UNED.

[18] Meneses, J. C. (1995). Secuencia de enseñanza sobre el electromagnetismo. Enseñanza de las Ciencias, 13(1), 36-45.
[19] Moreira, M. A. (1998). Modelos mentales y aprendizaje de física en electricidad y magnetismo. Enseñanza de las Ciencias: revista de investigación y experiencias didácticas, 16(2), 289.

[20] Muñoz, M. (1996). Using graphical based sostware to aid in the understanding of electromagnetic field theory. Frontiers in Education Conference, 2, 863867.

[21] Mukhopadhyay, S. C. (2008). Development of mathcad aided education materials for teaching electromagnetics at undergraduate level at massey university. International Journal of Technology and Engineering Education, 5(1).

[22] Nagornov, C. R. (2). Animando la física partei: Propagación de ondas. Revista Argentina de Enseñanza de la Ingeniería, (3), 39-44.

[23] Sagnard, F. (2004). Educational graphical interfaces to learn about radiation and propagation of electromagnetic waves. IEEE Transactions on Education, 47, doi:10.1109/te.2004.825531.

[24] Sebastián Dormido, F. E. (2005). Adding interactivity to existing simulink models using easy java simulations.en ieee (ed.). 44th IEEE Conference on Decision and Control, and the European Control Conference. Sevilla, España.

[25] Sevgi, L. (2008). A new electromagnetic engineering program and teaching via virtual tools. Progress in Electromagnetic Engineering Reserach, 6, 205-225. Sevilla, España.

[26] Spyrellis, G. K. E. P. J. P. N. (2009). 3d visualization types in multimedia applications for science learning: A case study for 8th grade students in greece. Computers and Education, 52, doi:10.1016/j.compedu.2008. 09.011.

[27] Vélez, B. E. (2014). Análisis de la enseñanza y el aprendizaje del eelectromagnetismo en el nivel tecnológico y universitario. REVISTA ENTRE CIENCIA E INGENIERÍA, 12.

[28] Vieites, B. V. (2007). Actividades manipulativas para el aprendizaje de la física. (l. c. organización de estados iberoamericanos para la educación, ed.). Revista Iberoamericana de Educación, 7(42). ISSN: 16815653. 